\title{
Study on the Effect of Feedback on the Learning and Performance of Motor Skills based on Error Results
}

\author{
Fan Zhang \\ Department of Police Skills and Tactics \\ Nanjing Forest Police College \\ No. 28 Wen Lan Road, Qixia District \\ Nanjing, JIANGSU 210023 China \\ zhangfan@nfpc.edu.cn
}

\begin{abstract}
The purpose of this study was to explore the effects of the error direction judgment on the motor performance and learning in the non-self-controlled conditions. 20 normal college students were randomly divided into Group WK and Group XD, with 10 participants ( 5 men and women in each group), and the force control motor of slider pushing and slipping was the experimental work. The correct practice of judging error direction, the error value of motor performance and the subjective estimation of motor error value are higher than the error in the error direction judgment. Under the condition of non-self-control, the error in the error direction of the motor learners and the external feedback information may have no effect on the accuracy and stability of performance and learning.
\end{abstract}

Keywords-Feedback; Motor skill learning; Motor performance; Error judgment

\section{INTRODUCTION}

In the past, the study of self-controlled feedback in motor learning focused on the benefit of motivational stimulation, learning strategies and psychological needs for motor learning. However, how is the effect of motor learning with the feedback information function inferred from the error estimates (refers to the process when people have not yet received extrinsic feedback after the execution of the motor, they subjectively compare the intrinsic feedback information produced by the motor with the correct motor in mind, to define the error of the motor execution), the actual performance and the feedback request. At present, there is a lack of corresponding research. It is necessary to explore the mechanism of information function in the process of self-controlled feedback affecting motor learning. In view of this, this study is based on the feedback information function, and takes the result of error detection of movement performance as the feedback basis. Under the situation of non-self-control, it examines the influence of information function provided by extrinsic feedback on movement learning after learners perform motor the error of which cannot be correctly judged, and further examines the movement performance after error judgment, with a view to providing scientific reference for the training and teaching of courses related to movement skills.

\section{RESEARCH OBJECTS AND METHODS}

\section{A. Research Objects}

20 healthy college students were chosen as subjects (10 men and 10 women) with the average age of 20.1 \pm 2.2 . They were randomly divided into error direction misjudgment feedback group (WK Group) and relative frequency control group (XD Group); each group had 10 participants (5 men and 5 women in the group).

\section{B. Research Methods}

1) Research Content:

Based on the feedback of self-control in the past study, when learners take the initiative to ask for feedback, the error rate of misjudging the error direction is not as high as that not asking for feedback. Then the question is whether it is helpful for learners to ask for feedback information after performing motor. In addition, previous studies have found that in the context of self-controlled feedback, learners' actions to with feedback request often have a higher error rate in judging the direction of error. Therefore, the following three research questions are put forward.

Question 1: What is the impact of motor performance and motor learning by providing feedback after the error direction is misjudged?

Question 2: Whether the motor' s error direction is judged correctly or not, is there any difference in the movement accuracy and the subjective estimate error value?

Question 3:Does the learner's actual motor performance differ from the subjective estimate?

Through providing feedback to solve the motor accuracy according to the result of the error direction judgment, the following hypothesis is put forward :

Hypothesis 1: During the exercise period and in the retention test as well as the transfer test, the $\mathrm{AE}$ value and $\mathrm{VE}$ value of the WK group show significant difference with those of the XD group.

Hypothesis 2: For WK group in the practice stage, AE value with feedback on the motor performance and $\mathrm{AE}$ value of subjective estimate are significantly lower than those without feedback. 
Hypothesis 3: AE value of subjective estimate in WK group is significantly lower than the $\mathrm{AE}$ value of actual motor.

a) Calculation Method of Absolute Error (AE)

The absolute error is calculated by the mean difference between the actual motor performance and the target value, and the absolute value is taken so it is not considered whether the motor performance is more or less than the direction of the target [1]. Only the difference between the motor and the target is tested. This method is used to evaluate the accuracy of the motor (Schmidt \& Lee, 2011). The calculation formula of absolute error is in (1):

$$
\mathrm{AE}=\Sigma\left|\mathrm{x}_{\mathrm{i}}-\mathrm{T}\right| / \mathrm{n}
$$

Note: $\mathrm{x}_{\mathrm{i}}=$ Result after motor execution, $\mathrm{T}=$ Target value of motor execution, $\mathrm{n}=$ Number of times for motor execution

\section{b) Calculation Method of Variation Error}

The variation error is to calculate the difference between the actual motor performance and the person's average motor performance, to indicate the difference between the result of the person's specific motor performance and the average performance, and the variation error is used evaluate the stability of the person's operation performance [2,3]. The formula for the variation error is in (2):

$$
\mathrm{VE}=\sqrt{\frac{\sum\left(\mathrm{x}_{\mathrm{i}}-\mathrm{M}\right)^{2}}{\mathrm{n}}}
$$

Note: $\mathrm{X}_{\mathrm{i}}=$ The result of a certain motor, $\mathrm{M}=$ The average of the results of an motor, $\mathrm{n}=$ Number of times for motor execution.

\section{2) Experimental Design:}

Based on the feedback of XD group, the subjects were assigned to WK Group and XD Group. After the experiment and demonstration, the participants conducted 3 familiar exercises, and the process did not cover the results of the motor. After each practice, the results of the data were learned. 3 min after the acquisition period, the exercises were carried out 60 time. On the 1th day, each subject was asked to carry out exercises 60 times ( $6 \times 10$ times), in which the researchers would provide different extrinsic feedback according to the group. 24 hours after the motor exercise, the retention test and the transfer test were carried out 12 times respectively. During the test process, no extrinsic feedback information was provided.

a) Feedback Group with Misjudgment on Error Direction (WK Group)

After each exercise in the acquisition period, the participants of this group reported the misjudged value for the exercise result orally. If the error value estimated by the participants and the error value of the actual practice result belonged to different directions of the practice target value, the researcher would provide the actual practice result of this exercise and carry out the next exercise after 10s. If the error value estimated by the participants and the error value of the actual practice result were on the same side of the practice target, the researcher would not provide the result of this exercise and would carry out the next exercise after $10 \mathrm{~s}$.

\section{b) Control Group of Relative Frequency (XD Group)}

After each exercise in the acquisition period, the participants of this group reported the misjudged value for the exercise result orally. The group's extrinsic feedback was arranged to match with the WK Group, to control the equivalence of the two sets of relative frequencies. 24 hours after the acquisition period, the retention test and the transfer test were carried out 12 times respectively. During the test process, no extrinsic feedback information was provided.

\section{3) Experiment:}

\section{a) Experimental Equipment}

The experiment equipment included seamless stainlesssteel smooth platform, screen, cylindrical slider of hard thermoplastic resin $(8 \mathrm{~cm}$ in diameter, $4.5 \mathrm{~cm}$ in height), notebook, code table, gauge, laser pen and right-angle gauge. The experimental site was quiet and nobody was present except the researchers and the subjects. The participants stood at the beginning of the platform to perform the experiment, and the researchers were on the side of the platform's sliding area, where the researchers collected each motor result and the subject's misjudged value.

\section{b) Experimental Methods}

The subjects, standing at the beginning of the platform, holding the slider in their hands, applied force to the slider at the zone of motor and release the slider in front of the screen. The extension line $300 \mathrm{~cm}$ away from the screen was used as the stop target, and the the screen covering the slide area of the slider during the motor performance. After each practice, the subjects were asked to evaluate the error value of the motor execution result, and to inform the experimenter orally. The researchers also provided the result of the motor orally. The results of the subjects' evaluation and the results of the researchers' information were presented in the way of positive/negative+ values, such as "positive 12", for the result of exceeding the target value of $12 \mathrm{~cm}$.

\section{c) Experimental Principle}

This experiment used intermittent power to control motor, so the result information would not be provided when the motor performers were applying force to it. The motor result can be obtained when the slider completely stopped, so the motor cannot be corrected according to the feedback during the motor execution, but could only be taken as the basis of the next motor, instead of correcting the performance of the current motor $[3,4]$. The layout of the experimental equipment covers the screen. Therefore, in the absence of visual information, after the implementation of the error detection and error estimation for each motor, motor performers must make judgment based on the body feeling and dynamic awareness of internal feedback.

\section{4) Experiment Flow:}

\section{a) Acquisition period (acquisition phase)}

Each participant in the two groups was given a total of 60 exercises divided into 6 intervals, 10 exercises per interval, and 3 minutes between intervals. 


\section{b) Retention Test and Transfer Test}

24 hours after the end of the acquisition period, 12 times of exercises with the goal of $300 \mathrm{~cm}$ for the retention test was carried out as well as the transfer test with the goal of $250 \mathrm{~cm}$. No extrinsic feedback was provided during the process of retention test and transfer testing. Data was collected in order to avoid warm-up decrement [5,6]. The first 2 exercises were eliminated for analysis.

\section{5) Statistical processing and analysis:}

SPSS 19.0 was used for statistical processing, and the results were expressed by means standard deviation $(\bar{X} \pm \mathrm{SD})$. The independent variable provided the feedback method, because the variable was the accuracy of the motor, the stability and the error range. The accuracy of the motor is evaluated by $\mathrm{AE}$, and the motor stability is evaluated by VE. The following statistical methods were proposed to validate the research hypothesis. Two factor variance analysis with the mixed design of 2 (Group) x 6 (interval) was carried out to validate hypothesis 1 , for comparison later. The hypothesis 2 was verified by 2 (feedback) x 3 (interval) two-factor variance analysis, and 2 (subjective/objective) x3 (interval) two-factor variance analysis was made to verify the hypothesis 3 . Statistically the significant level was $P<0.05$ and the extremely significant level was for $P<0.01$.

\section{RESULTS AND ANALYSIS}

\section{A. Difference in frequency offeedback}

WK group provided feedback. When the learners performed the motor, before the extrinsic feedback was provided, the subjective error estimation (error value) was made, and then the researcher compared it with the actual motor performance of the error value. When the subjective and objective error direction was inconsistent, the researchers provided extrinsic feedback information, or no extrinsic feedback was to be provided for this motor. The frequency of actual feedback in each interval of the study was $24.2 \%$ for 1 th interval, $25.8 \%$ for 2 nd interval, $40 \%$ for 3 rd interval, $45.8 \%$ for 4 th interval, $33.3 \%$ for 5 th interval and $31.7 \%$ for 6 th interval. The average frequency of all interval feedback was $33.5 \%$.

Since the specific learner in a certain interval correctly judged the error direction 10 times, there was no extrinsic feedback provided. So the 2 intervals were combined to be 1 data points, forming a total of 3 intervals. Through the single factor analysis of variance, the feedback frequency $(25 \%$ for 1th interval, $42.9 \%$ for 2 nd interval and $32.5 \%$ for 3rd interval) was provided. The results showed that there were statistically significant differences $((\mathrm{F}(2,22)=7.51, \mathrm{p}<.05, \eta 2=.11$, medium effect size), in which Interval 2' $\mathrm{s}$ frequency was significantly higher than that of Interval 1 and Interval 3, while Interval 1 was not significantly different from Interval 3.

\section{B. The Difference between motor performance and motor learning}

1) $A E$ :

Acquisition period: The results showed that the intermotor between the groups and the intervals was not statistically significant $(\mathrm{F}(2.8,110)=1.87, \mathrm{p}>.05, \eta 2=.03$, small effect size). The main effect of groups had no statistically significant difference $(\mathrm{F}(1,22)=.25, \mathrm{p}>.05, \eta 2=.0)$. The main effect of intervals had statistically significant difference $(\mathrm{F}(2.8$, $110)=46.39, \quad \mathrm{p}<.05, \eta 2=.63$, large effect size). After comparison, it was found that the absolute error value of motor performance had a significant reduction with the practice interval, among which the 5th and 6th interval showed a flat tendency.

Retention test and transfer tests: The results showed there were no statistically significant differences between the groups, for the retention test $(\mathrm{t}(22)=.54, \mathrm{p}>.05, \mathrm{~d}=.22$, small effect size) and for the transfer test $(\mathrm{t}(22)=1.06, \mathrm{p}>.05, \mathrm{~d}=.43$, small effect size).

\section{2) $V E$ :}

Acquisition period: The results showed that there were statistically significant differences between groups and intervals $(\mathrm{F}(2.59,110)=.44, \mathrm{p}>.05, \eta 2=.01)$. The main effect of groups had no statistically significant difference $(F(1,22)=$ $1.55, \mathrm{p}>.05, \eta 2=.02)$. The main effect of intervals had statistically significant difference $(\mathrm{F}(2.59,110)=14.25, \mathrm{p}<.05$, $\eta 2=.26$, large effect size). The VE value of motor performance had a significant reduction with the practice interval. After comparison, it was found that the VE value of Interval 1 was significantly higher than the Interval 2 6, and that the VE value of Interval 5 and 6 was significantly lower than that of Interval 1 4

Retention test and transfer tests: The results showed there were no statistically significant differences between the groups, for the retention test $(\mathrm{t}(22)=1.12, \mathrm{p}>.05, \mathrm{~d}=.46$, small effect size) and for the transfer test $(\mathrm{t}(22)=.89, \mathrm{p}>.05, \mathrm{~d}=.36$, small effect size).

All in all, 1) During the acquisition period, in the performance accuracy of $\mathrm{AE}$ and $\mathrm{VE}$, there was no difference between WK group and XD group, which did not support Hypothesis 1.2 ) There was no difference in the performance accuracy between WK group and XD group in the retention and transfer test, for VE representing the accuracy of performance and for VE representing the stability of performance, which did not support Hypothesis 2.

\section{Difference between the motor performance with and without feedback}

1) motor Performance with and without feedback

The results of the actual motor analysis showed that the motor performance with and without feedback as well as two factor interactions did not reach statistically significant differences $(\mathrm{F}(2,44)=.82, \mathrm{p}>.05, \eta 2=.0)$. For the factor of with and without feedback, it was found that $\mathrm{AE}$ values reached statistically significant differences $(F(1,22)=14.52$, $p$ $<.05, \eta 2=.11$, medium effect size), of which the average AE value (32.92) of motor with feedback was significantly lower than the average AE value of the exercise without feedback. There were significant differences in the performance of the exercises with and without feedback in each interval, and the $\mathrm{AE}$ values of the motor with feedback in 3 intervals (the absolute error value of each interval was 42.5, 28.7 and27.5, respectively), were all significantly lower than those without 
feedback (the absolute error value of each interval 53.1, 40.2 and 34.9 , respectively).

The main effect of interval factor had statistically significant difference $(\mathrm{F}(2,44)=50.23, \mathrm{p}<.05, \eta 2=.22$, large effect size). After comparison, it was found that the absolute error value of the motor performance was significantly reduced with the practice interval (the average of each interval is 47.8 , 34.6 and 31.2, respectively). Through comparing the AE values of the exercises with feedback in each interval, it was found that the average of 1 th interval was significantly larger than that of 2nd interval and the 3rd interval, while the 2nd and 3rd intervals had no statistically significant difference (the average absolute error values of each interval was 42.6, 28.7 and 27.5, respectively). Through comparing the $\mathrm{AE}$ values of the exercises without feedback in each interval, it was found that the average of 1th interval average (53.1) was significantly larger than the average of the 2nd interval (40.4) and the 3rd interval (34.9), while there was no statistically significant difference between 2nd and 3rd interval.

2) Subjectively estimated motor performance with and without feedback

After testing, the motor performance with and without feedback as well as two factor interactions did not reach statistically significant differences $(F(2,44)=2.46, p>.05$, $\eta 2=.01)$. For the factor without feedback, it was found that $\mathrm{AE}$ value reached statistically significant differences $(\mathrm{F}(1$, $22)=35.71, p<.05, \eta 2=.29$, large effect size), the average AE with feedback on the estimated exercise performance (13.06) (the absolute error value each of interval was 12.6, 13.7 and 12.8 , respectively) in each interval was significantly lower than those without feedback (22.72) (the absolute error value of each interval is $24.5,22.7$ and 20.9, respectively). The main effect of interval factor had no statistically significant difference $(\mathrm{F}(2,44)=2.02, \mathrm{p}>.05, \eta 2=.01)$, and the average absolute error value of each interval was $18.58,18.22$, and 16.87 respectively.

3) Subjectively estimated error value with feedback and $A E$ value of actual motor Performance

After examination, subjective and objective error value as well as interval factor interaction reached statistically significant difference $(F(1.6,44)=18.75, \mathrm{p}<.05, \eta 2=.04$, small effect size), and factors of subjective and objective error also had statistically significant difference $(F(1,22)=71.25, p$ $<.05, \eta 2=.33$, large effect size), in which the AE value (13.06) of the subjectively estimated motor performance (the absolute error value of each interval was 12.6, 13.7 and 12.8, respectively; each interval in the group did not have the significant difference) was significantly lower than the actual $\mathrm{AE}$ value (32.92) (the absolute error value of each interval was42.6, 28.7 and 27.5, respectively; each interval in the group was significantly different, and Interval 1 was significantly greater than Interval 2 and Interval 3; there was no significant difference between Interval 2 and Interval 3). Among three intervals, there were statistically significant differences between groups. The actual error value was significantly greater than the estimated error value, and the main effect of interval factor had statistically significant difference $(F(2,44)=$ 2.02, $\mathrm{p}<.05, \eta 2=.04$, small effect size). The average absolute error in each interval was 27.59, 21.2 and 20.17; Interval 1 was significantly different with Interval 2 and Interval 3; there were no significant difference between Interval 2 and Interval 3.

4) Subjectively estimated error value without feedback and $A E$ value of actual motor performance

After examination, subjective and objective error value as well as interval factor interaction reached statistically significant difference $(\mathrm{F}(2,44)=15.48, \mathrm{p}<.05, \eta 2=.03$, small effect size), and factors of subjective and objective error also had statistically significant difference $(F(1,33)=106.24$, $p$ $<.05, \eta 2=.34$, large effect size). The interval factor had statistically significant difference $(F(2,44)=33.3, p<.05$, $\eta 2=.07$, medium effect size). After comparison, it was found that, in terms of subjective and objective error value, the average $\mathrm{AE}$ value of motor performance without feedback was 22.72 (the absolute error values of each interval was 24.5, 22.7 and 20.9, respectively; there were significant differences between the intervals. Interval 1 was significantly larger than Interval 3. There were no significant differences between Interval 2, Interval 1 and Interval 3). The actual AE value was 42.79 (the absolute error values of each interval was 53.1, 40.4 and 34.9 , respectively; there were significant differences among the 3 intervals). The subjectively estimated $\mathrm{AE}$ value in three intervals was significantly lower than the $\mathrm{AE}$ value of actual performance. In terms of the actual $\mathrm{AE}$ value, the averages of 3 intervals have a downward trend with the performance practice, and there are statistically significant differences between intervals, but there were no significant differences between the intervals in the subjectively incorrectly estimated $\mathrm{AE}$ value.

After analyzing the WK group's acquisition period, the $\mathrm{AE}$ value with feedback on the motor performance was significantly lower than that without feedback, so the result supported the Hypothesis 2. In addition, the result of comparison between subjective estimation and the actual objective performance of WK group supported Hypothesis 3 . The statistic results showed that the estimated error value was significantly lower than that of actual motor performance, with or without feedback provided.

\section{CONCLUSION}

Under the condition of non-self control, when the extrinsic feedback information is provided for the learner to judge errors in error direction, there is no effect on the accuracy and stability of the motor performance and learning. Error direction is used to judge the correct practice. The error value of the motor performance and the subjectively estimated error value of motor performance are all higher than that of the misjudged error direction; that is to say, the accuracy of actual motor performance with misjudged error direction is higher than those with correctly judged error direction. It is the same with the subjectively estimated motor performance. The absolute error value of the learner's actual motor is higher than that of the subjective estimate; that is, the learner has an overestimation of self-expression, regardless of whether the learner correctly judges the error direction or not. 


\section{ACKNOWLEDGMENT}

This work was supported in part by the Project of the Fundamental Research Funds for the Central Universities under Grant LGZD201805, in part by Jiangsu University Philosophy and Social Sciences Research Fund Project under Grant 2017SJB0590, in part by the 13th Five-Year plan project of Jiangsu Education Science under Grant C-c/2018/01/11, in part by Nanjing Forest police College Teaching Reform Project under Grant ZD18104, in part by the Project of China Postdoctoral Science Foundation under Grant 2017M611849, in part by Jiangsu Qing Lan Project under Grant 2017, and in part by Pre-research project of Nanjing Forest police College under Grant LGY201603.

\section{REFERENCES}

[1] Vlaskamp. C, "Physical and occupational therapists' perceptions of motor control, motor development, and motor learning in the support of people with intellectual disabilities," Clinical Nutrition, vol. 6, pp. 201203, March 2012.

[2] Tore. P. A. D., Schiavo, R., \& D' Isanto. T, “Physical education, motor control and motor learning: theoretical paradigms and teaching practices from kindergarten to high school," Journal of Physical Education \& Sport, vol. 16, pp. 1293-1297, April 2016.

[3] Alessandro, Cristiano, et al, "Motor Control and Learning Theories. Emerging Therapies in Neurorehabilitation," Springer International Publishing, 2016.

[4] Schmidt, Richard A, and T. D. Lee, "Motor control and learning : a behavioral emphasis," Human Kinetics, 1999.

[5] Bund. A, Wiemeyer. J, Bund. D. A, "Self-controlled Learning of a Complex Motor Skill: Effects of the Learners' Preferences on Performance and Self-efficacy," Journal of Human Movement Studies, vol. 47, pp. 215-236, March 2004.

[6] Liu. J, Fu. H. J, Chen. S, et al., "The Effect of Provided and SelfRequested Knowledge of Performance on Acquisition and Transfer Performance of an Open Sport Skill in College Students," Journal of the National Cancer Institute, vol. 105, pp. 1111-1122, July 2014. 